\title{
Efficient strategy evaluation to control the COVID-19 pandemic
}

\author{
Rafael Gonzalez \\ Universidad Mayor \\ Moya Pablo \\ University of Chile \\ Eduardo Bringa \\ University of Mendoza \\ Gonzalo Bacigalupe \\ University of Massachusetts Boston \\ Muriel Ramirez-Santana ( $\nabla$ mramirezs@ucn.cl) \\ Catholic University of the North \\ Miguel Kiwi \\ University of Chile
}

\section{Research Article}

Keywords: COVID-19, control, Efficient strategy evaluation

Posted Date: March 17th, 2021

DOI: https://doi.org/10.21203/rs.3.rs-305039/v1

License: (c) (i) This work is licensed under a Creative Commons Attribution 4.0 International License.

Read Full License 

19 the implementation of many non-pharmaceutical 20 interventions $(\mathrm{NPI})^{2-6}$. Detailed analysis of these 21 policies utilized by different countries have been 22 completed 7 . One of the main challenges is to estimate 23 the threat posed by the pandemic to plan effective 24 interventions 89 that are specific and have a precise 25 timing $10 \mid 11$.

26 There are many NPIs but, in general, reducing human ${ }_{27}$ interactions is considered crucial ${ }^{11}$. The Test, Trace, ${ }_{28}$ and Isolate (TTI) strategy has been shown to contribute 29 to decreasing the epidemic growth $[517$ when the number 30 of new cases is low and feasible to trace. Otherwise, 31 confinement is an alternative of control when the number 32 of new cases is threatening to overload health services, 33 together with an enormous social and economic impact ${ }^{12}$. ${ }_{34}$ No matter which one, the NPI are measures aimed just 35 towards reducing the number of infections and reaching
36 the "ideal" of zero infected cases. Available data shows 37 that this aim alone is insufficient, since a measure that 38 succeeds in one country might not prove to be effective 39 in another. Quite often the data is unreliable ${ }^{13 \mid 14}$ due 40 to different methodologies used to collect and process ${ }_{41}$ the data, among other reasons (asymptomatic cases, lack ${ }_{42}$ of testing, and false-negative RT-PCR tests 15 . 20 . But, ${ }_{43}$ in spite of the latter, actions are needed. For that ${ }_{44}$ purpose, we put forward a model focused on identifying 45 the risk signals that the available data reveal. Ironically, 46 we use tools developed by Isaac Newton during a long ${ }_{47}$ past pandemic. To do so, we consider, in addition to 48 the number of cases and their time evolution, how the 49 increase or decrease of cases varies in time. In other 50 words, and in close analogy to the dynamics of motion, 51 we incorporate in our model the speed of change of the ${ }_{52}$ time evolution of the number of infections.

${ }_{53}$ At this point, it is essential to clarify our approach. ${ }_{54}$ Having zero contagion should not be taken literally; 
${ }_{55}$ COVID-19 will likely continue to circulate for a long ${ }_{56}$ time $^{21}$. However, while there are no effective treatments 57 or while massive vaccination is not reached, it is ${ }_{58}$ reasonable to seek to cut the contagion. And this can be a ${ }_{59}$ lesson learned for future pandemics: seek to cut or reduce 60 the contagion while science works on safe solutions.



FIG. 1. Diagram to illustrate how the proposed performance variables are defined and calculated. The variables are the reduction rate $\rho$, the growth rate $G$, and the variation rate $V$.

${ }_{61}$ To control the pandemic, the WHO recommends 62 achieving ".. a decline of infections of at least $50 \%$ ${ }_{63}$ over a 3 -week period since the latest peak, and a ${ }_{64}$ continuous decline in the observed incidence of confirmed 65 and probable cases..."22]. Indeed, it is easier said than ${ }_{66}$ done and, therefore, we develop below precise data ${ }_{67}$ analysis to contribute quantitative criteria in line with ${ }_{68}$ the WHO recommendation. We start defining the seven${ }_{69}$ days reduction rate, illustrated in Fig. 1, as

$$
\rho(t)=\frac{N(t)-N_{\max }}{N(t)},
$$

70 where $t$ labels a specific day, $N(t)$ is the weekly average 71 number of cases on date $t$ and $N_{\max }$ corresponds to a 72 maximum of daily cases during those seven days. This 73 way, when the number of infections decreases, $\rho<0$.

74 The growth rate $G$ instead is the ratio of the average 75 weekly increase and the minimum of infections, that is

$$
G(t)=\frac{N(t)-N_{\min }}{N_{\min }},
$$

76 where $N_{\min }$ corresponds to a minimum of daily cases 77 during those seven days. Since $\rho$ is computed relative to 78 a maximum and $G$ to a minimum, the former turns out 79 to be significantly smaller numerically, a fact that has to so be kept in mind when comparing their magnitudes.

81 The variation $V$ is defined as follows:

$$
V(t)=\frac{N(t)-N(t-7)}{N(t-7)},
$$

82 and all these time variations can be associated with a 8з corresponding "velocity." For example, we define the ${ }_{84}$ reduction velocity as

$$
v_{\rho}(t)=\frac{d \rho(t)}{d t}
$$

${ }_{85}$ where the derivative is computed numerically with ${ }_{86}$ discrete differences. In the same way we define the 87 growth and variation velocities.

${ }_{88}$ Several different indicators have to be incorporated 89 in the analysis to enhance the probability of effectively 90 controlling COVID-19, but essentially what is required is ${ }_{91}$ to reduce the number of cases as fast as feasible. When a 92 new contagion focus is found, TTI or equivalent measures 93 may be applied, but if they do not yield prompt results 94 (i.e., a significant reduction within 1-2 weeks), it means 95 that the strategy is falling short. Therefore, it is useful 96 to analyze the time variation of the variables above, i.e., ${ }_{97}$ the time variation of the reduction.

98 In order to demonstrate the usefulness of these 99 variables, next we use them to examine three countries, 100 with significantly different COVID-19 evolution, as 101 prototypes: Vietnam, Australia, and the UK. We start 102 with Vietnam, a successful crisis management example ${ }_{103}$ with a solid NPI that kept the total number of cases at 10415 per million and the total number of deaths at 0.4 per 105 million (February 2021). Close examination of Fig. $2 \mathrm{a}$ 106 reveals that since March 2020, they have been able to 107 keep a strong reduction rate by reacting promptly to 108 the rise of infections. In fact, they managed to keep the 109 reduction rate $\rho$, as defined in Eq. 1. in negative territory 110 except for a slight increment in the second half of July 1112020 , followed by rapid reaction, but which nevertheless 112 led to a peak of just 0.4 cases per million a day.

113 We now turn our attention to Australia, illustrated in ${ }_{114}$ Fig. 2b, which was hit by COVID-19 in early March. 115 The reaction was swift but the weekly average number 116 of cases reached $\sim 15$ per million by the end of March 117 and was contained by the end of April. However, by 118 mid-June $\rho$ became positive, and the reaction was not 119 speedy enough to prevent a second wave which peaked 120 at 21 cases per million in mid-August and extended to ${ }_{121}$ early October, but has been under control since.

What happened in the United Kingdom, where the ${ }_{123}$ pandemic has not yet been controlled, is also worthwhile 124 to examine critically. The reduction rate was already in ${ }_{125}$ positive terrain $\rho>0$ in early March, and the number of ${ }_{126}$ cases per million $N(t)$ grew to $\sim 100$ per million by April, ${ }_{127}$ without a decrease until the end of June. Unfortunately, 128 in August $\rho$ grew again indicating that a second wave was 129 approaching, which became quite serious by November 130 and has not been brought under control. We write these ${ }_{131}$ lines (February 2021) after reaching almost 800 daily new 132 cases per million. This confirms that the implemented 133 strategies did not succeed.

${ }_{134}$ Having perused what happened in Vietnam (a success 135 story), Australia (initial success followed by a second 136 wave), and the United Kingdom (where control of the 
137 situation was lost), we can now try to infer some general 195 required.

138 behavior about epidemic management.

139 The data examined above suggests that, if one pays 140 close attention to the reduction rate, two general 141 conclusions can be drawn. The first inference is that 142 keeping the reduction rate $\rho$ constant is not sufficient; 143 instead, one has to aim towards a steady decrease. 144 Also, speedy action is required as soon as the reduction 145 rate $\rho>0$. Below we verify how general these 146 prescriptions are by examination of additional available 147 data. All of the data used below was obtained from 148 ourworldindata.org 23 .

149 New Zealand has been singled out as a success story 150 in handling COVID. We now present a detailed study 151 of what happened there, as illustrated in Fig. 3. in the 152 light of the methodology put forward above. There 153 was a significant outbreak in March, which prompted a 154 vigorous reaction that reduced spectacularly, within a 155 month, the number of cases, which then remained low 156 for nearly two months. Thereafter, every time there was 157 an outbreak, it was contained in a matter of days, as 158 occurred with the August and October peaks. Each time 159 a new outbreak, no matter how small, became manifest, 160 there was a swift response, mainly implementing TTI, 161 with a strong effort to trace contacts, locate the infected 162 and put them in isolation. A similar strategy was also ${ }_{163}$ followed in Iceland (See Fig. S4 in S.M.). In Fig. 3 a, 164 we observe that rapid action is advisable as soon as the 165 variation rate $V>0$, and always keeping $\rho<0$ for as long 166 as possible. The dynamics are more evident when Fig. $3 \mathrm{~b}$ 167 is inspected, since the mid-August action and reaction 168 are more pronounced when the velocity is plotted vs. $t$. ${ }_{169}$ These conclusions are reinforced by Fig. 3p, where we 170 show that it is enough to follow the reduction rate and 171 growth velocity to analyze trends.

172 In summary, we compute three variables and focus on 173 their time variation: case growth, its variation, and the 174 evolution speed. In particular, New Zealand's growth 175 velocity is in general significantly larger than the other 176 two variables, and allows for a rapid pinpointing of 177 the instant case reduction beginning. Therefore, the 178 reduction rate is central to our analysis, and furthermore 179 allows to warn authorities and the population with a 180 simple message: if reduction stops, there is trouble ahead.

${ }_{181}$ We present the analysis for more than 50 other 182 countries in the Supplementary Information. The data 183 plotted in Fig. 4 for all those countries is intended to 184 show that our analysis is consistent with the worldwide 185 evolution of COVID-19. We plot the average weekly 186 reduction rates $\rho$ vs. the logarithm of the average number ${ }_{187}$ of cases in Fig. $4 \mathrm{a}$ and the logarithm of the average 188 number of deaths in Fig. $4 \mathrm{~b}$. The countries that have 189 fared best are located top left while the worst performers 190 are mostly found bottom right. Again it is quite apparent 191 that a quick response and a prolonged time negative 192 reduction rate $(\rho<0)$ yield favorable results. Reduction 193 alone does not predict performance well enough for 194 some countries, like Japan, and additional indicators are
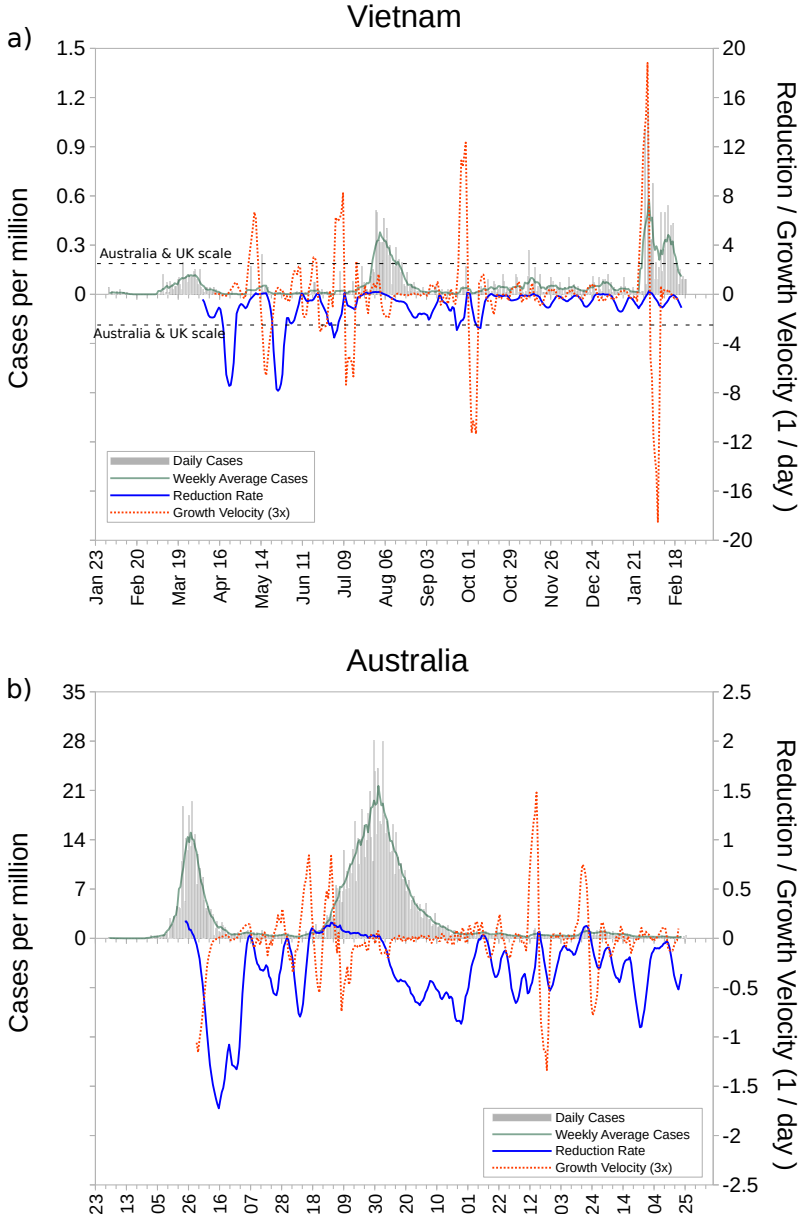

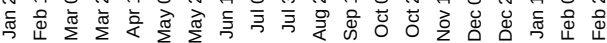

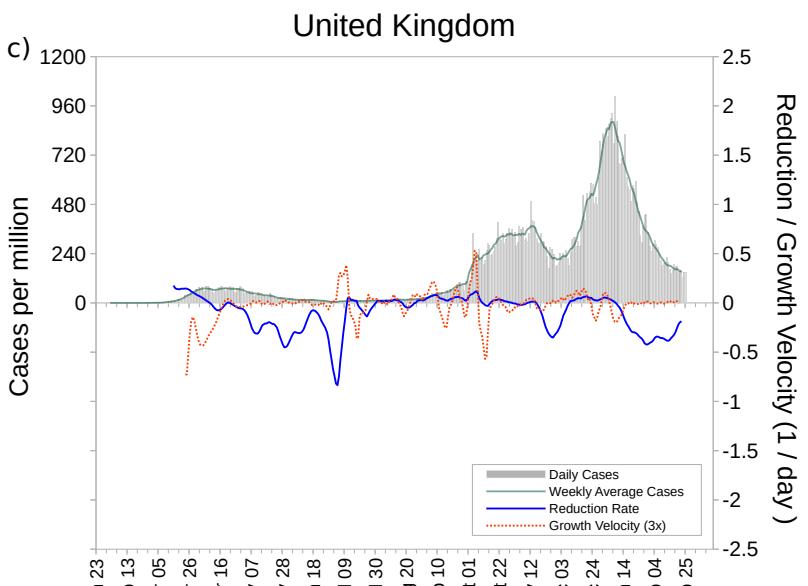

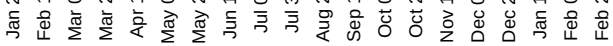

FIG. 2. Illustration of the COVID19 evolution in three countries with quite different progressions. Vietnam, a success story. Australia, which has faced a couple of serious episodes, but which lately have been overcome. The United Kingdom has not performed well, and at this time, is still facing a rather difficult moment. 
a)

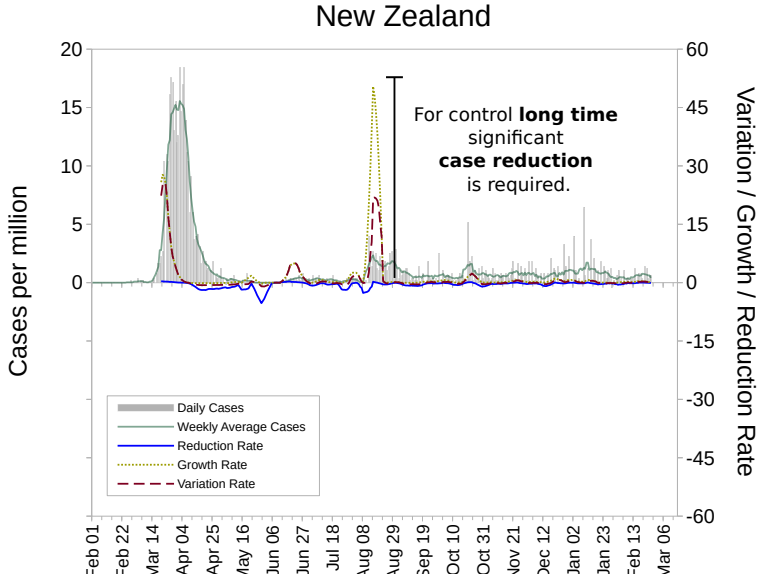

b)

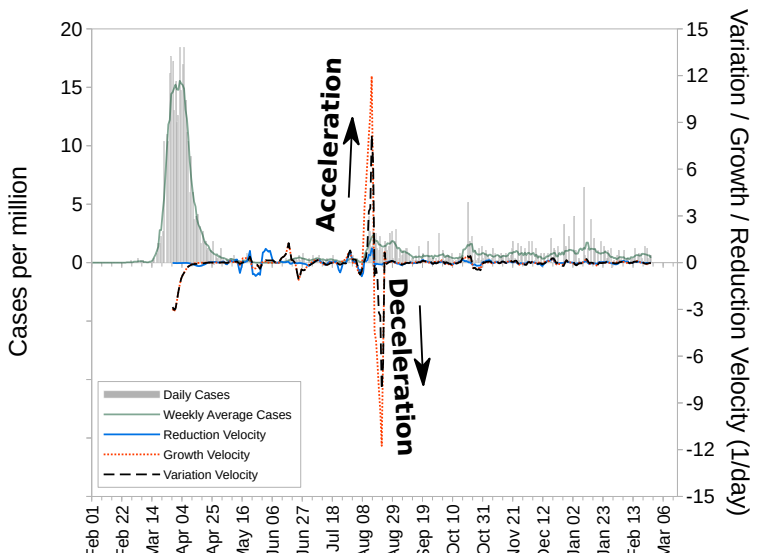

c)

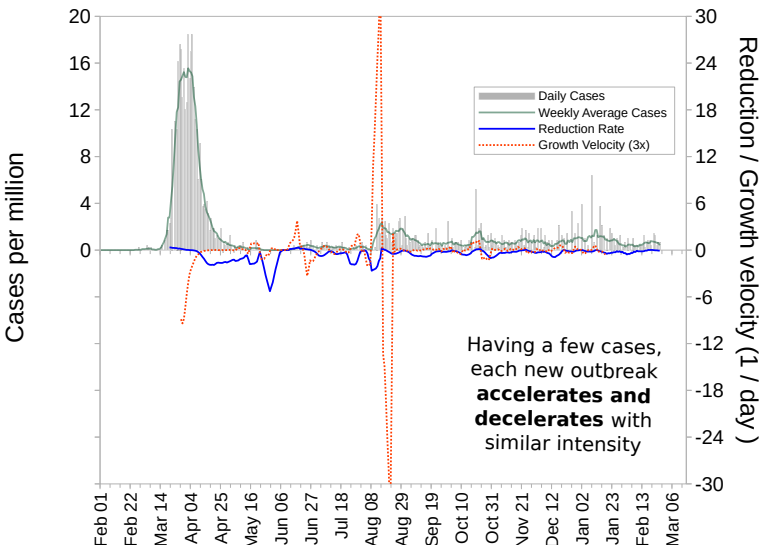

FIG. 3. Time evolution of the variables. The date $t=0$ is chosen throughout as the day the particular country exceeds 100 infections. a) Weekly averages of the number of cases, variation, reduction and growth rates as a function of time. Notice that the growth rate is many times larger than the reduction rate. b) Number of cases, their growth velocity, reduction velocity and variation velocity vs. time. c) Number of cases, their reduction and growth velocity vs. time.

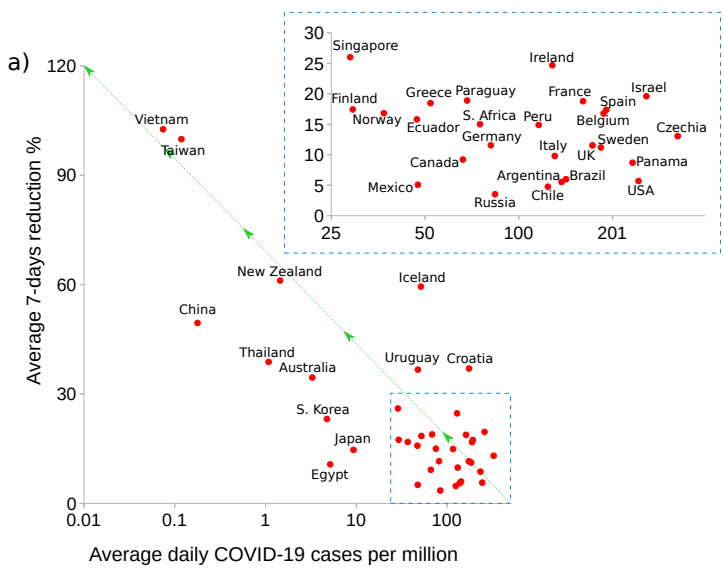

b)
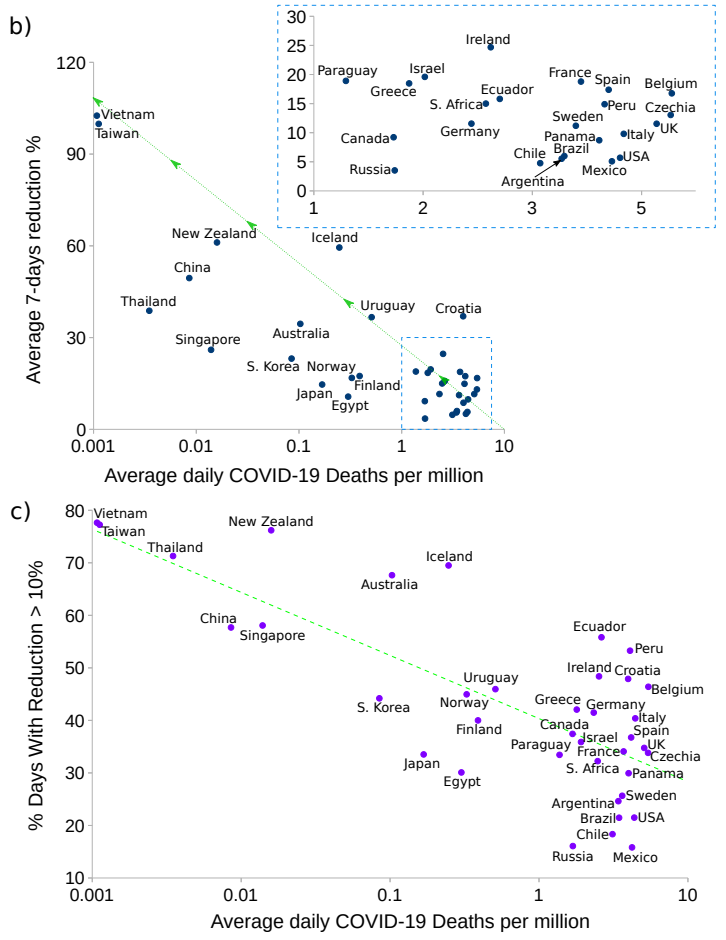

FIG. 4. Data of many countries in scatter plots. a) Weekly average percentage of case reduction vs. average daily COVID-19 cases per million. Countries that have done well are consistently found upper left. b) Weekly average of the number of days COVID is reduced vs. average daily COVID19 deaths per million; again we observe a similar country distribution as in a). c) Number of days where the reduction rate was large than $10 \%$ as vs. average daily COVID-19 deaths per million. There is a clear difference between the countries that have successfully controlled infections (upper left) and the ones that have not done so well (lower right). For each country, the daily average was calculated since they exceeded 100 accumulated cases.

196 It is important to point out that Fig. 4 corresponds to 197 a snapshot taken at a precise moment and varies in time; 198 however, the average reduction goes slowly enough to 199 portray more than 40 countries in just one figure. These 200 plots do not constitute a final verdict, but they allow to 
201 spot the cases that strongly deviate from the norm. They 237 indicators in the paper display country-wide behaviors, 202 could be related to poor tracing ${ }^{24 \mid 25}$, different ways to 238 but they can also be applied at a more local level, 203 keep statistics and/or sub-reporting.

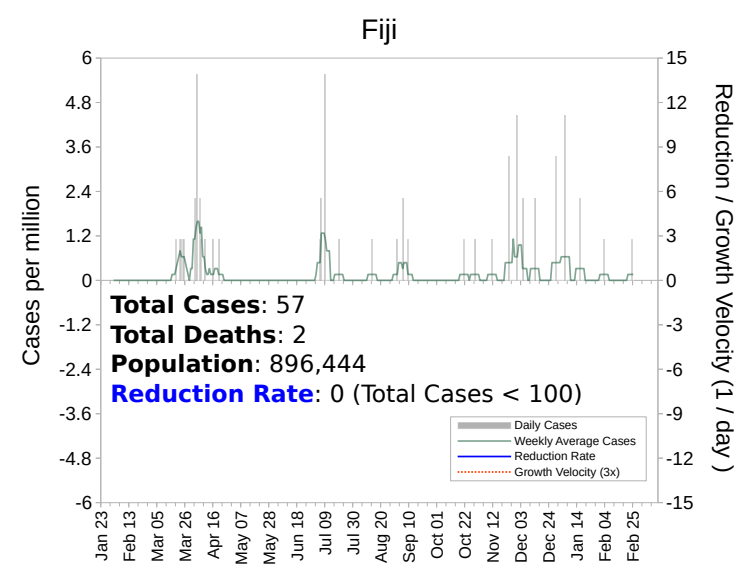
239 and might also contribute to better management of 240 the epidemic when only a sector of the population is ${ }_{241}$ considered, for instance based on age or income.

242

\section{METHODS}

243 The data for all countries was obtained from ${ }_{244}$ ourworldindata.org ${ }^{23}$ on February 26, 2021, including 245 deaths and population data. As in the first days of the 246 epidemic the data can vary a lot, all the calculations 247 of variations were made once the country reported more 248 than 100 accumulated cases. Similarly, given that the 249 data can vary significantly from day to day, we calculate 250 the seven days moving centered case average. The rest of 251 the variation averages are obtained as the rolling seven 252 days average.

FIG. 5. Fiji, the country with the best results among the more ${ }^{253}$ The reduction, growth, and variation rate were than 50 we analyzed. Since we computed the reduction rate ${ }_{254}$ computed from the average daily cases. We define the when the number of total cases exceeds 100 it is not included ${ }_{255}$ daily rate of change of the variation, named as velocity, above. Fiji has informed of only 57 cases and 2 deaths due to COVID-19 by February 25, 2021. 256 with finite differences. For
257 velocity is computed as follows:

204 Finally, we must mention Fiji in Fig. 5 , a country that ${ }_{205}$ in February 2021 has not exceeded 100 total cases, so 206 we do not apply the variation analysis of other countries. ${ }_{207}$ However, it is evident that with a population close to 900 208 thousand inhabitants, 63 total cases per million and 2.2 209 total deaths per million shows it as one of the countries 210 with the best response of all those we analyzed.

$$
v_{\rho}(t)=\frac{d \rho(t)}{d t} \approx \frac{\rho(t-2 t)-8 \rho(t-1)+8 \rho(t+1)-\rho(t+2)}{\Delta 12}
$$

258 where $\rho$ is the reduction rate and $t$ is the date. In this 259 case we used a 4 day central finite differece.

260 During the first weeks of the epidemic the velocity data 211 Mitigating the impact of a pandemic and achieving ${ }_{261}$ can be quite noisy, so we decided to calculate the velocity 212 zero contagion is a primordial public health goal. ${ }^{262}$ on the average of the reduction, growth, or variation (as ${ }_{213}$ Developing sound methodological tools to alert policy ${ }_{263}$ appropriate) during the first three weeks after reaching 214 makers is essential to implement NPIs while the vaccine is 264100 cases. During the rest of the days it is calculated 215 still under development, fabrication, and administration. ${ }_{265}$ directly on the variations without averaging. We present 216 Moreover, there is uncertainty about the impact of the 266 the averaged speed every seven days in the curves, so 217 vaccine stopping the contagion before reaching herd 267 that they look smoother.

${ }_{218}$ immunity or preventing further reactivation of the virus ${ }_{268}$ To calculate the total variation of Fig. 4 of the 219 or new waves of contagion.

269 manuscript we added all the daily reductions without 220 We have presented performance indicators applicable 270 averaging, and they were divided by the total number 221 to any NPI: reduction, growth, and variation rates (or 271 of days since the 100 accumulated cases were exceeded. 222 velocities). These indicators complement other existing 272 The reduction rate was multiplied by 100 and expressed 223 ones, like the effective reproduction number $R_{0}$, and 273 as a positive number for clarity.

${ }_{224}$ complex epidemic models 26127 . In fact, the application ${ }_{274}$ Finally, it is worth mentioning that for 7 countriegs we 225 of these indicators to a large number of countries allows 275 found some anomalous data that caused the calculation ${ }_{226}$ early warning about a likely epidemic growth, even when 276 of the variations to be altered. This is because the 227 other indicators fail to do so. The "universality" of these ${ }_{277}$ countries made data corrections during the course of 228 indicators could signal not only the need to strengthen ${ }_{278}$ the emergency. As the spirit of this work is to keep 229 certain NPI in order to improve the epidemic control, 279 it simple, we decided to replace those values with the 230 but could also help with the performance evaluation of 280 average number of cases during the previous 7 days. For ${ }_{231}$ the vaccination campaigns that are already taking place ${ }_{281}$ France this had to be done in many times, some were less 232 in some countries.

282 than 7 days apart. Therefore, this procedure was followed ${ }_{233}$ To reach control of the epidemic, independent of the ${ }_{283}$ successively in time. Some special days for France were ${ }_{234}$ NPI implemented and of the progress in the vaccination ${ }_{284}$ October 25 and 26, as well as between November 2 and ${ }_{235}$ process, significant case reduction for extended periods 2854 , and what we did was to replace each day with the 236 is necessary, keeping the growth rate under zero. The 286 average of those days. 
287 All in all we tried our best to modify the data as little ${ }_{292}$ ${ }_{288}$ as possible. The total cases we used the total are the ones 289 reported on February 26, 2021. In the Supplementary 290 Information we include all the modified data, marked in 291 yellow, to clarify the modifications we made.

\section{ACKNOWLEDGMENTS}

Supported by ANID Chile through Fondecyt grants 294 Nos. 11180557, 1191351, and Center for the Development 295 of Nanoscience and and Nanotechnology, CEDENNA 296 AFB180001. (2020). (2020). mramirezs@ucn.cl

Dyani Lewis, "Superspreading drives the covid pandemic- 35 and could help to tame it." Nature 590, 544-546 (2021). ${ }_{352}$

2 Solomon Hsiang, Daniel Allen, Sébastien Annan-Phan, 353 Kendon Bell, Ian Bolliger, Trinetta Chong, Hannah 354 Druckenmiller, Luna Yue Huang, Andrew Hultgren, Emma 355 Krasovich, et al., "The effect of large-scale anti-contagion 356 policies on the covid-19 pandemic," Nature 584, 262-267 357

3 Sen Pei, Sasikiran Kandula, and Jeffrey Shaman, 359 "Differential effects of intervention timing on covid-19 360 spread in the united states," Science Advances 6 (2020). ${ }_{361}$

4 Neil J. Rowan and Rafael A. Moral, "Disposable face 362 masks and reusable face coverings as non-pharmaceutical 363 interventions (npis) to prevent transmission of sars-cov- 364 2 variants that cause coronavirus disease (covid-19): 365 Role of new sustainable npi design innovations and 366 predictive mathematical modelling," Science of The Total 367 Environment 772, 145530 (2021).

5 Adam J Kucharski, Petra Klepac, Andrew J K Conlan, ${ }^{36}$ Stephen M Kissler, Maria L Tang, Hannah Fry, Julia R 370 Gog, W John Edmunds, Jon C Emery, Graham Medley, 371 James D Munday, Timothy W Russell, Quentin J 372 Leclerc, Charlie Diamond, Simon R Procter, Amy Gimma, 373 Fiona Yueqian Sun, Hamish P Gibbs, Alicia Rosello, 374 Kevin van Zandvoort, Stéphane Hué, Sophie R Meakin, 375 Arminder K Deol, Gwen Knight, Thibaut Jombart, 376 Anna M Foss, Nikos I Bosse, Katherine E Atkins, Billy J ${ }_{377}$ Quilty, Rachel Lowe, Kiesha Prem, Stefan Flasche, Carl 378 A B Pearson, Rein M G J Houben, Emily S Nightingale, 379 Akira Endo, Damien C Tully, Yang Liu, Julian Villabona- 380 Arenas, Kathleen O'Reilly, Sebastian Funk, Rosalind M 381 Eggo, Mark Jit, Eleanor M Rees, Joel Hellewell, Samuel 382 Clifford, Christopher I Jarvis, Sam Abbott, Megan 383 Auzenbergs, Nicholas G Davies, and David Simons, 384 "Effectiveness of isolation, testing, contact tracing, and 385 physical distancing on reducing transmission of sars-cov- 386 2 in different settings: a mathematical modelling study," 387 The Lancet Infectious Diseases 20, 1151-1160 (2020). ${ }^{6}$ Nils Haug, Lukas Geyrhofer, Alessandro Londei, Elma 389 Dervic, Amélie Desvars-Larrive, Vittorio Loreto, Beate 390 Pinior, Stefan Thurner, and Peter Klimek, "Ranking 391 the effectiveness of worldwide covid-19 government 392 interventions," Nature human behaviour 4, 1303-1312 393

7 Amy Dighe, Lorenzo Cattarino, Gina Cuomo-Dannenburg, 395 Janetta Skarp, Natsuko Imai, Sangeeta Bhatia, Katy AM 396 Gaythorpe, Kylie EC Ainslie, Marc Baguelin, Samir 397 Bhatt, et al., "Response to covid-19 in south korea 398 and implications for lifting stringent interventions," BMC 399 medicine 18, 1-12 (2020).

8 Megan O'Driscoll, Ribeiro Dos Santos, G. Wang, Lin 401 Wang, Derek A. T. Cummings, Andrew S. Azman, Juliette
Paireau, Arnaud Fontanet, Simon Cauchemez, and Henrik Salje, "Age-specific mortality and immunity patterns of SARS-CoV-2," Nature 590, 140-145 (2021).

9 G. Pullano, Di Domenico, and C.E. et al. L., Sabbatini, "Underdetection of covid-19 cases in france threatens epidemic control." Nature 590, 134-139 (2021).

10 Jeffery Shaman, "An estimation of undetected covid cases in france," Nature 590, 38-39 (2021).

11 Akihiro Nishi, George Dewey, Akira Endo, Sophia Neman, Sage K. Iwamoto, Michael Y. Ni, Yusuke Tsugawa, Georgios Iosifidis, Justin D. Smith, and Sean D. Young, "Network interventions for managing the covid-19 pandemic and sustaining economy," PNAS 117, 3028530294 (2020).

12 Isaac Yen-Hao Chu, Prima Alam, Heidi J Larson, and Leesa Lin, "Social consequences of mass quarantine during epidemics: a systematic review with implications for the COVID-19 response," Journal of Travel Medicine $\mathbf{2 7}$ (2020).

13 Ian McCulloh, Kevin Kiernan, and Trevor Kent, "Inferring true covid19 infection rates from deaths," Frontiers in Big Data 3, 37 (2020).

14 Peter Lloyd-Sherlock, Lucas Sempe, Martin McKee, and Aravinda Guntupalli, "Problems of Data Availability and Quality for COVID-19 and Older People in Low- and Middle-Income Countries," The Gerontologist 61, 141-144 (2020).

15 Steven Woloshin, Neeraj Patel, and Aaron S. Kesselheim, "False negative tests for sars-cov-2 infection - challenges and implications," New England Journal of Medicine $\mathbf{3 8 3}$, e38 (2020).

16 Rita Christiane Baron, Lorenz Risch, Myriam Weber, Sarah Thiel, Kirsten Grossmann, Nadia Wohlwend, Thomas Lung, Dorothea Hillmann, Michael Ritzler, Susanna Bigler, Konrad Egli, Francesca Ferrara, Thomas Bodmer, Mauro Imperiali, Sonja Heer, Harald Renz, Lukas Flatz, Philipp Kohler, Pietro Vernazza, Christian R. Kahlert, Matthias Paprotny, and Martin Risch, "Frequency of serological non-responders and falsenegative rt-pcr results in sars-cov-2 testing: a populationbased study," Clinical Chemistry and Laboratory Medicine (CCLM) 58, $2131-2140$ (2020).

17 Chunqin Long, Huaxiang Xu, Qinglin Shen, Xianghai Zhang, Bing Fan, Chuanhong Wang, Bingliang Zeng, Zicong Li, Xiaofen Li, and Honglu Li, "Diagnosis of the coronavirus disease (covid-19): rrt-pcr or ct?" European Journal of Radiology , 108961 (2020).

${ }^{18}$ Lauren M Kucirka, Stephen A Lauer, Oliver Laeyendecker, Denali Boon, and Justin Lessler, "Variation in falsenegative rate of reverse transcriptase polymerase chain reaction-based sars-cov-2 tests by time since exposure," Annals of Internal Medicine 173, 262-267 (2020). 
${ }_{402}{ }^{19}$ Michael J. Mina and Kristian G. Andersen, "Covid-19 ${ }_{419}$ 403 testing: One size does not fit all," Science 371, 126-127 420 $404 \quad$ (2021).

${ }_{405}{ }^{20}$ Daniel B. Larremore, Bryan Wilder, Evan Lester, Soraya ${ }_{422}$

406 Shehata, James M. Burke, James A. Hay, Milind Tambe, ${ }_{423}$

${ }_{407}$ Michael J. Mina, and Roy Parker, "Test sensitivity is ${ }_{424}$ 408 secondary to frequency and turnaround time for covid-19 425 409 screening," Science Advances 7 (2021).

${ }_{410} 21$ "Covid-19 is a marathon, not a sprint (editorial)," Nature 427 411 human behaviour 5, 1 (2021).

${ }_{412}{ }^{22}$ World Health Organization, Public health criteria to adjust ${ }_{429}$ 413 public health and social measures in the context of COVID- 430 ${ }_{414}$ 19: annex to considerations in adjusting public health and ${ }_{431}$ 415 social measures in the context of COVID-19, 12 May 2020, 43 416 Technical documents (2020).

${ }_{417}{ }^{23}$ Joe Hasell, Edouard Mathieu, Diana Beltekian, Bobbie ${ }_{434}$ 418 Macdonald, Charlie Giattino, Esteban Ortiz-Ospina, Max 435
Roser, and Hannah Ritchie, "A cross-country database of covid-19 testing," Scientific data 7, 1-7 (2020).

${ }^{24}$ Dyani Lewis, "Why many countries failed at covid contacttracing-but some got it right." Nature 588, 384-387 (2020).

25 Simon Munzert, Peter Selb, Lukas F. Stoetzer, and Will Lowe, "Tracking and promoting the usage of a covid-19 contact tracing app," Nature human behaviour 5, 247-255 (2021).

26 Damon Centola, "Considering network interventions," Proceedings of the National Academy of Sciences 117, 32833-32835 (2020).

27 Serina Chang, Emma Pierson, Pang Wei Koh, Jaline Gerardin, Beth Redbird, David Grusky, and Jure Leskovec, "Mobility network models of covid-19 explain inequities and inform reopening," Nature 589, 82-87 (2021) 


\section{Figures}

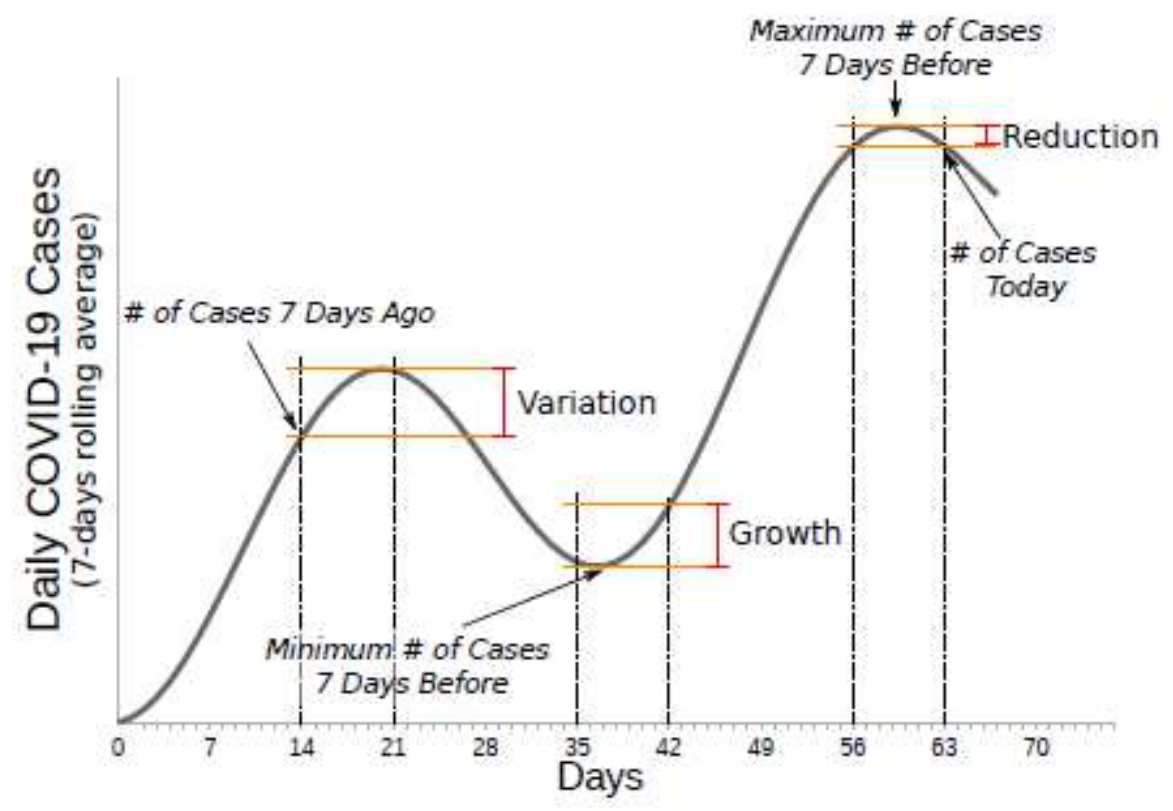

\section{Figure 1}

Diagram to illustrate how the proposed performance variables are dened and calculated. The variables are the reduction rate $p$, the growth rate $G$, and the variation rate $V$. 

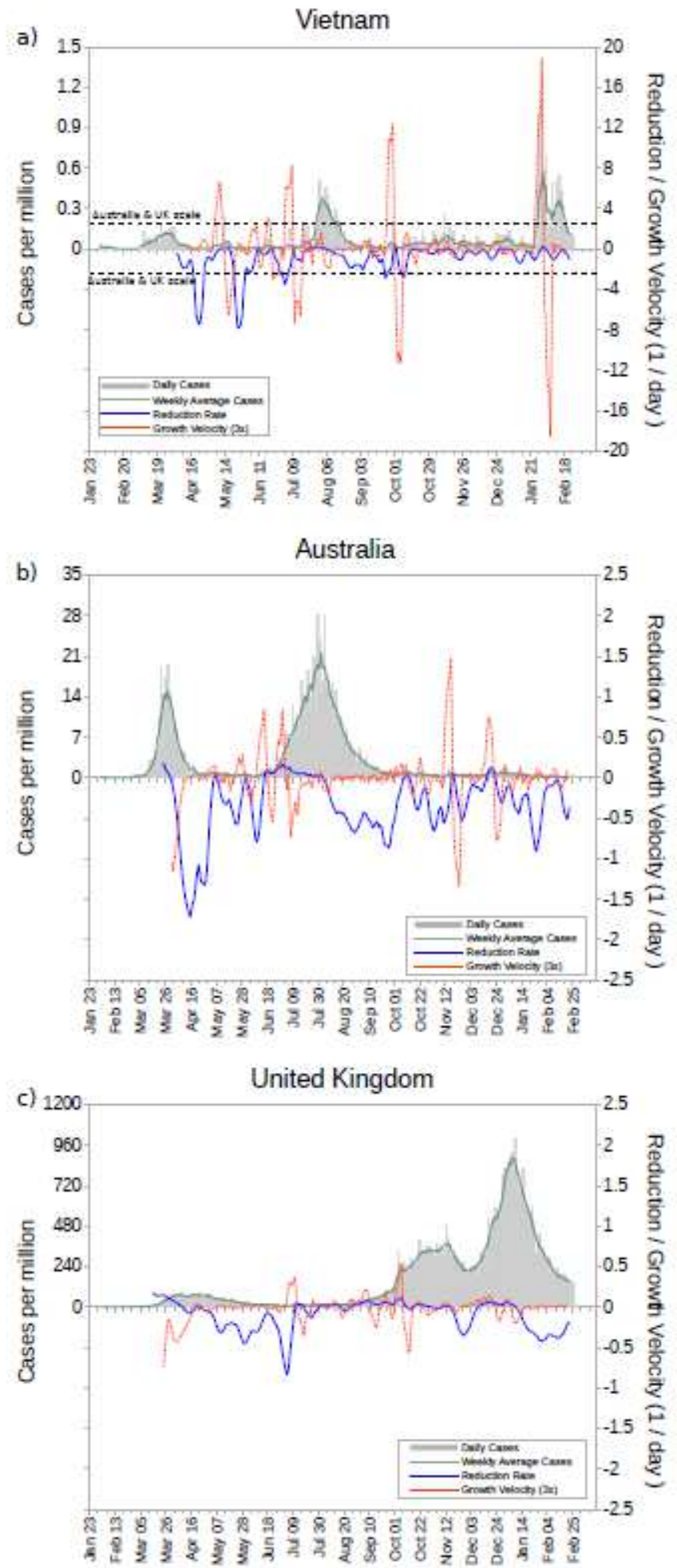

Figure 2

Illustration of the COVID19 evolution in three countries with quite different progressions. Vietnam, a success story. Australia, which has faced a couple of serious episodes, but which lately have been overcome. The United Kingdom has not performed well, and at this time, is still facing a rather difficult moment. 
a)

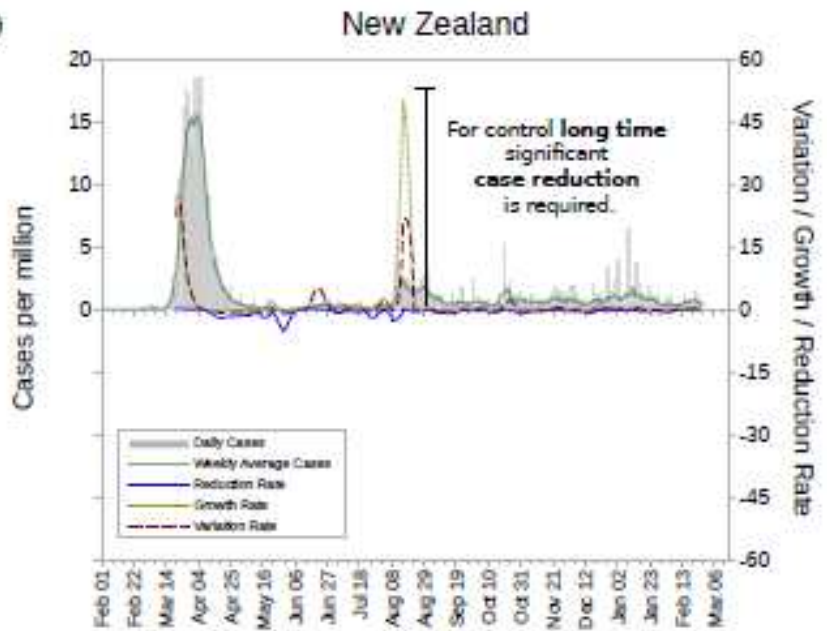

b)

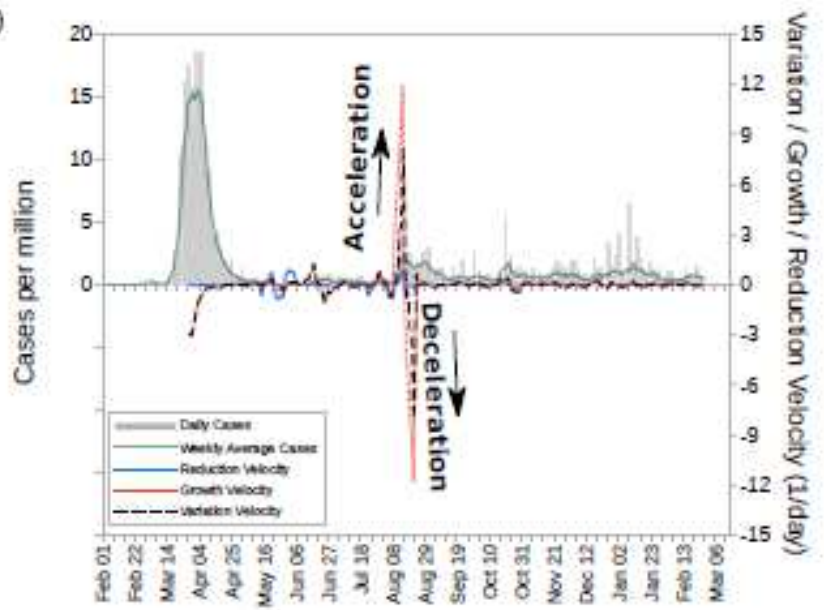

c)

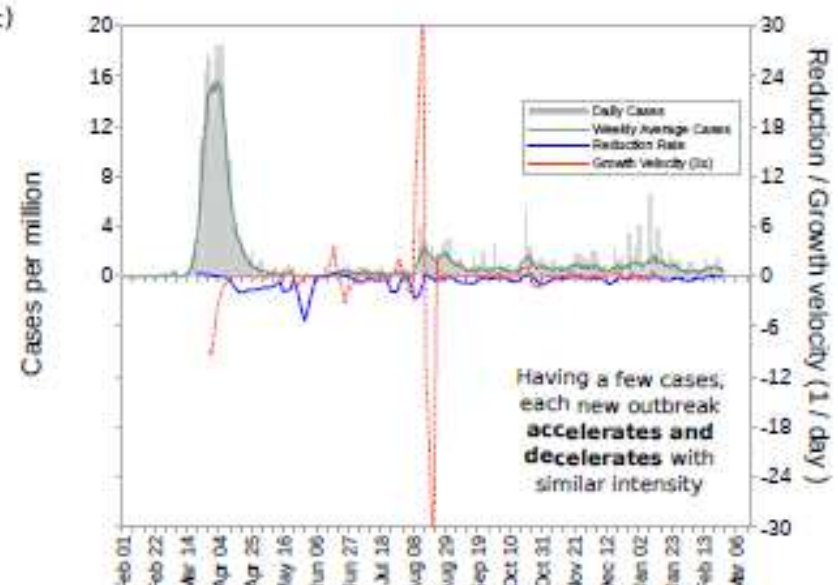

Figure 3

Time evolution of the variables. The date $t=0$ is chosen throughout as the day the particular country exceeds 100 infections. a) Weekly averages of the number of cases, variation, reduction and growth rates as a function of time. Notice that the growth rate is many times larger than the reduction rate. b) Number of cases, their growth velocity, reduction velocity and variation velocity vs. time. c) Number of cases, their reduction and growth velocity vs. time. 

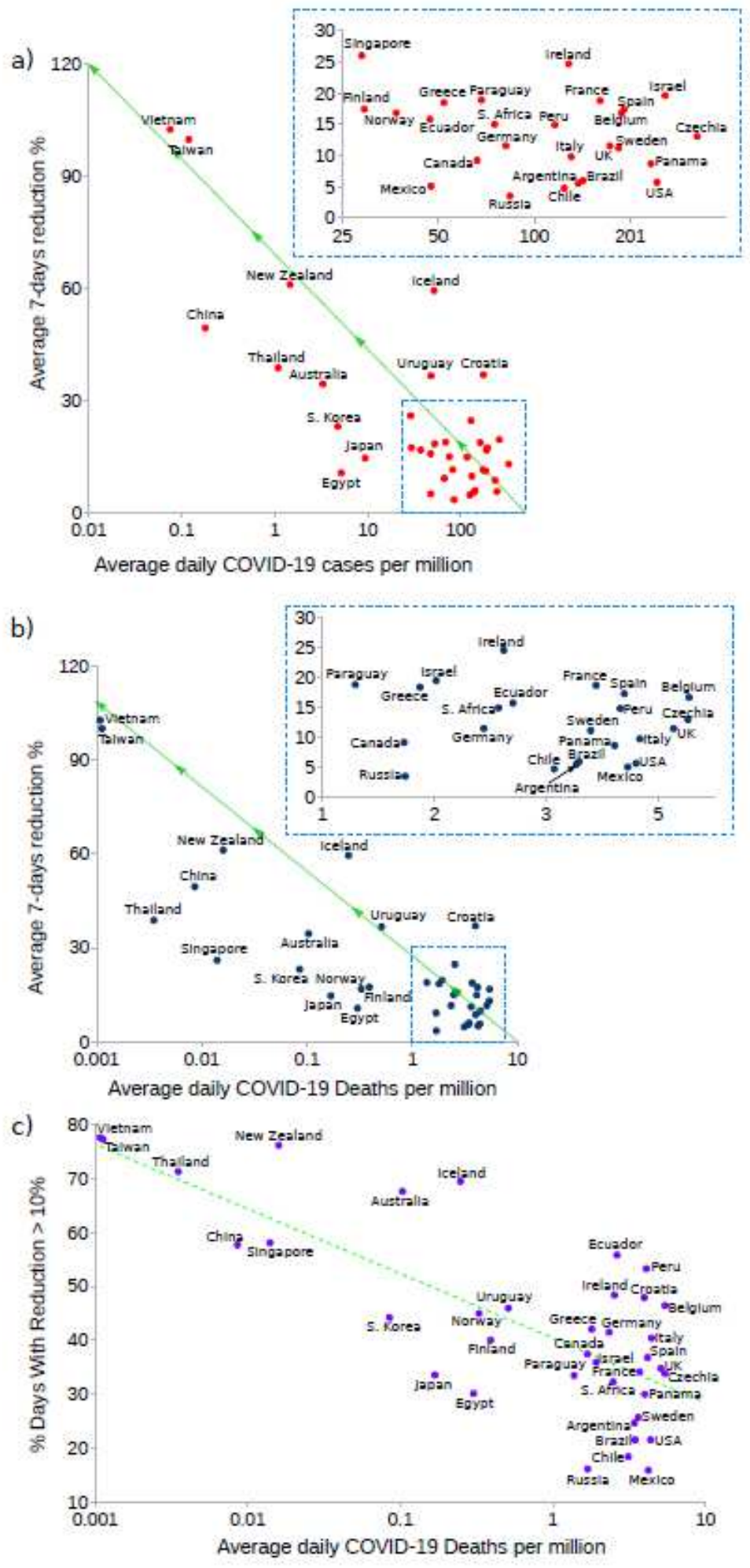

\section{Figure 4}

percentage of case reduction vs. average daily COVID-19 cases per million. Countries that have done well are consistently found upper left. b) Weekly average of the number of days COVID is reduced vs. average daily COVID- 19 deaths per million; again we observe a similar country distribution as in a). c) Number of days where the reduction rate was large than $10 \%$ as vs. average daily COVID-19 deaths per million. There is a clear difference between the countries that have successfully controlled infections (upper left) and 
the ones that have not done so well (lower right). For each country, the daily average was calculated since they exceeded 100 accumulated cases.

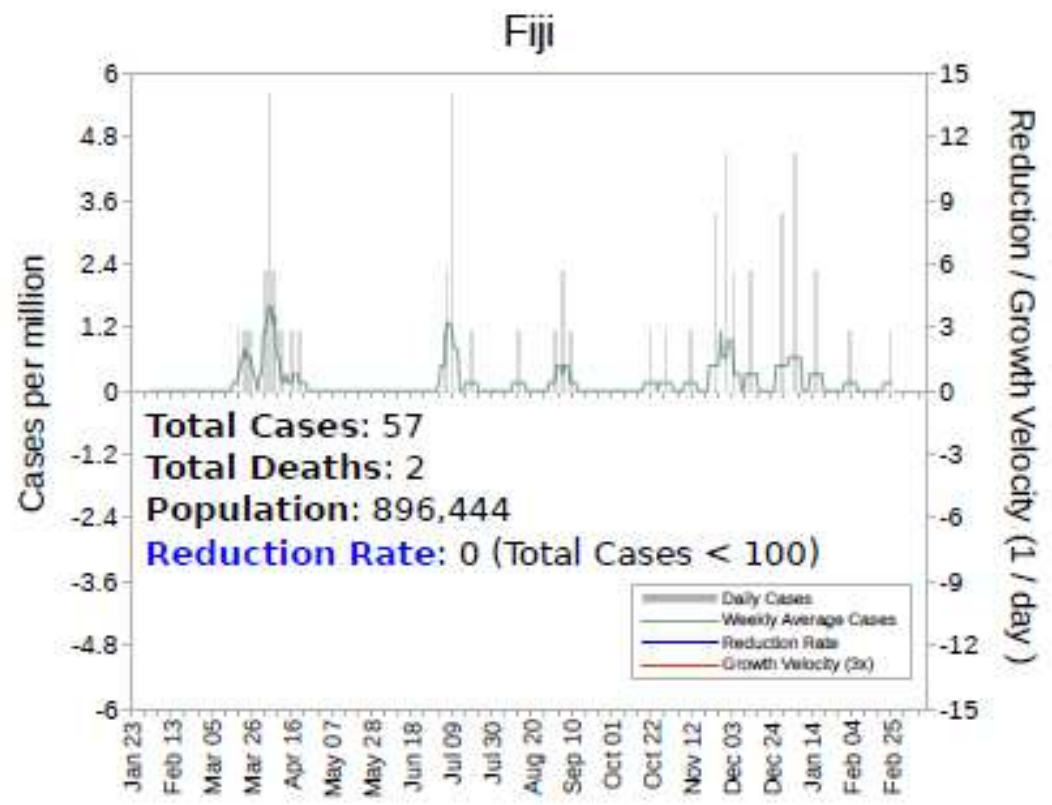

Figure 5

Fiji, the country with the best results among the more than 50 we analyzed. Since we computed the reduction rate when the number of total cases exceeds 100 it is not included above. Fiji has informed of only 57 cases and 2 deaths due to COVID-19 by February 25, 2021.

\section{Supplementary Files}

This is a list of supplementary files associated with this preprint. Click to download.

- SuppEfficientstrategyevaluationtocontrol.pdf 